\title{
Vibrational spectra of halide-water dimers: Insights on ion hydration from full-dimensional quantum calculations on...
}

Article in The Journal of Chemical Physics · March 2018

DOI: $10.1063 / 1.5005540$

CITATIONS

0

4 authors, including:

Xiao-Gang Wang

Queen's University

87 PUBLICATIONS 1,843 CITATIONS

SEE PROFILE
READS

10

Tucker Carrington

Queen's University

231 PUBLICATIONS 7,096 CITATIONS

SEE PROFILE 


\section{Vibrational Spectra of Halide-Water Dimers: Insights on Ion Hydration from}

Full-Dimensional Quantum Calculations on Many-Body Potential Energy Surfaces

Pushp Bajaj, ${ }^{1}$ Xiao-Gang Wang, ${ }^{2}$ Tucker Carrington Jr., ${ }^{2}$ and Francesco Paesani, a)

1) Department of Chemistry and Biochemistry, University of California, San Diego, La Jolla, California 92093, U.S.A.

${ }^{2)}$ Chemistry Department, Queen's University, Kingston, Ontario KYL3N6, Canada

3) Department of Chemistry and Biochemistry, Materials Science and Engineering, and San Diego Supercomputer Center, University of California, San Diego, La Jolla, California 92093, U.S.A. 
Full-dimensional vibrational spectra are calculated for both $\mathrm{X}^{-}\left(\mathrm{H}_{2} \mathrm{O}\right)$ and $\mathrm{X}^{-}\left(\mathrm{D}_{2} \mathrm{O}\right)$ dimers (X $=\mathrm{F}, \mathrm{Cl}, \mathrm{Br}, \mathrm{I})$ at the quantum-mechanical level. The calculations are carried out on two sets of recently developed potential energy functions (PEFs), namely TTM-nrg and MB-nrg, using the symmetry-adapted Lanczos algorithm with a product basis set including all six vibrational coordinates. Although both TTM-nrg and MB-nrg PEFs are derived from CCSD(T)-F12 data obtained in the complete basis set limit, they differ in how many-body effects are represented at short range. Specifically, while both models describe long-range interactions through the combination of two-body dispersion and many-body classical electrostatics, the relatively simple Born-Mayer functions employed in the TTM-nrg PEFs to represent short-range interactions are replaced in the MB-nrg PEFs by permutationally invariant polynomials to achieve chemical accuracy. For all dimers, the MB-nrg vibrational spectra are in close agreement with the available experimental data, correctly reproducing anharmonic and nuclear quantum effects. In contrast, the vibrational frequencies calculated with the TTM-nrg PEFs exhibit significant deviations from the experimental values. The comparison between the TTM-nrg and MB-nrg results thus reinforces the notion that an accurate representation of both short-range interactions associated with electron density overlap and long-range many-body electrostatic interactions is necessary for a correct description of hydration phenomena at the molecular level.

Keywords: ion-water interactions, ionic clusters, vibrational spectroscopy

a) Electronic mail: fpaesani@ucsd.edu 


\section{INTRODUCTION}

A molecular-level understanding of halide-water interactions is key to characterizing the properties of complex electrolyte solutions that often mediate fundamental chemical, biological, and environmental processes. In this context, vibrational spectroscopy has emerged as a powerful tool to investigate structural and dynamical properties of ionic systems from the gas phase to the condensed phase. The assignment of different spectral features is, however, complicated by the fluctuating nature of the underlying hydrogen-bond network, which often results in a continuum of distinct structural motifs that vary as a function of system size, thermodynamic conditions, and chemical environment.

Recent advances in experimental techniques and theoretical methods have made possible to probe the hydration properties of small ions at the molecular level. Numerous studies have focused on determining the relative stability and structural properties of halide-water clusters through the analysis of the corresponding vibrational spectra, which have provided fundamental insights into the nature of the underlying molecular interactions. ${ }^{1-4}$ Halide- $^{-}$ water dimers, in particular, have been the subject of much interest since they serve as prototypical hydrogen-bonded complexes that exhibit large amplitude motion. In addition, since the potential energy surface (PES) of the halide-water dimers display two equivalent global minima with $\mathrm{C}_{s}$ symmetry separated by a $\mathrm{C}_{2 v}$ saddle point along the in-plane water rocking motion, the intermolecular vibrational levels may split due to quantum tunneling. ${ }^{5-7}$ This makes the theoretical assignment of the different absorption peaks observed in the experimental spectra particularly challenging, requiring both an accurate PES and a rigorous treatment of the nuclear motion at the quantum-mechanical level.

Due to its high proton affinity and, thus, relatively strong basic character, fluoride can establish strong hydrogen bonds with water, which are manifested in large redshifts of the $\mathrm{OH}$ stretching frequencies observed in the $\mathrm{F}^{-}\left(\mathrm{H}_{2} \mathrm{O}\right)$ vibrational spectra. The strength of the halide-water hydrogen bonds then decreases from fluoride to iodide. This is accompanied by a reduction of the tunneling barrier on the underlying PES and consequent increase of the splitting of the vibrational levels. Previous investigations of the vibrational spectra of halide-water dimers include experimental measurements using cold traps and argon tagging techniques combined with harmonic and reduced-dimensionality anharmonic frequencies calculated on PESs obtained at the MP2 level of theory. ${ }^{5,8,9}$ Large shifts in the hydrogen-bonded 
$\mathrm{OH}$ stretching frequencies and increases in peak intensities were observed in the vibrational spectra of $\mathrm{F}^{-}\left(\mathrm{H}_{2} \mathrm{O}\right)$, which can be attributed to the formation of strong hydrogen bonds and significant charge redistribution within the dimer.

Following the initial experimental measurements, six-dimensional calculations of the $\mathrm{Cl}^{-}\left(\mathrm{H}_{2} \mathrm{O}\right)$ vibrational spectrum were carried out within the MULTIMODE framework using full-dimensional potential energy and dipole moment surfaces fitted to $\operatorname{CCSD}(\mathrm{T})$ electronic structure data. ${ }^{10}$ More recently, anharmonic vibrational frequencies have been calculated for both $\mathrm{F}^{-}\left(\mathrm{H}_{2} \mathrm{O}\right)$ and $\mathrm{F}^{-}\left(\mathrm{D}_{2} \mathrm{O}\right)$ dimers using a variational method called GENIUSH and a semiglobal PES derived from CCSD(T)-F12a data. ${ }^{6}$ The GENIUSH method employs the internal coordinates and direct product DVR (discrete variable representation) basis. The acronym GENIUSH stands for general (GE) rovibrational code with numerical (N), internalcoodinate (I), user-specified (US) Hamiltonians (H). Fully anharmonic vibrational frequencies, including couplings between all vibrational modes, have been reported for $\mathrm{Cl}^{-}\left(\mathrm{H}_{2} \mathrm{O}\right)^{7}$. This calculation was done, with the same PES used in Ref. 10, using a product basis with DVR functions (D) for the stretches and spherical harmonic type functions (S) for the bends and a symmetry adapted Lanczos eigensolver (L). ${ }^{11,12}$ This method is referred to as DSL. The bend basis is appropriate for dealing with large amplitude bending motions. In all cases, good agreement was found between different theoretical predictions and the available experimental data.

In this study, we present a thorough analysis of the vibrational spectra of both $\mathrm{X}^{-}\left(\mathrm{H}_{2} \mathrm{O}\right)$ and $\mathrm{X}^{-}\left(\mathrm{D}_{2} \mathrm{O}\right)$ dimers, with $\mathrm{X}=\mathrm{F}, \mathrm{Cl}, \mathrm{Br}$, and $\mathrm{I}$, obtained from DSL calculations using two sets of ab initio halide-water PEFs (TTM-nrg for Thole-type model energy ${ }^{13}$ and MBnrg for many-body energy ${ }^{14}$ ) recently reported in the literature. We note here that the TTM-nrg PEFs were originally introduced with the i-TTM acronym. ${ }^{13}$ Since the TTMnrg and MB-nrg PEFs only differ in the way many-body (MB) effects are represented, the analysis of the vibrational spectra presented here provides fundamental insights into the relative importance of short- and long-range two-body (2B) interactions in determining the (inter)molecular dynamics. Furthermore, the direct comparison of the $\mathrm{X}^{-}\left(\mathrm{H}_{2} \mathrm{O}\right)$ and $\mathrm{X}^{-}\left(\mathrm{D}_{2} \mathrm{O}\right)$ spectra enables a quantitative assessment of the interplay between hydrogen-bond strength and nuclear quantum effects. 


\section{COMPUTATIONAL METHOD}

The vibrational frequencies of the $\mathrm{X}^{-}\left(\mathrm{H}_{2} \mathrm{O}\right)$ and $\mathrm{X}^{-}\left(\mathrm{D}_{2} \mathrm{O}\right)$ dimers were calculated using the method of Ref. 11,12, implemented in the RV4 code. ${ }^{15}$ It uses coordinates derived from two Radau vectors and a Jacobi vector, a basis of products of potential-optimized discrete variable functions(PODVR $)^{16,17}$ for the stretch coordinates and a spherical harmonic type basis for the bend coordinates. Potential energy integrals are computed with Gauss quadrature. Eigenvalues and eigenvectros are determined with the symmetry-adapted Lanczos (SAL) algorithm. ${ }^{18,19}$ Matrix-vector products are evaluated by doing sums sequentially. ${ }^{20,21}$ In this study, we shall refer to the method as the DSL method. Since a thorough description of the DSL method is reported in Ref. 7, only the computational details specific to the present study are given here. All calculations in this study are for $J=0$. Each basis function was

$$
f_{\alpha_{0}}\left(r_{0}\right) f_{\alpha_{1}}\left(r_{1}\right) f_{\alpha_{2}}\left(r_{2}\right) u_{l_{1} l_{2} m_{2} K}^{J M P}\left(\theta_{1}, \theta_{2}, \phi_{2} ; \alpha, \beta, \gamma\right)
$$

where, $f_{\alpha_{n}}\left(r_{n}\right)$ are the PODVR functions for the three stretching coordinates, and $u_{l_{1} l_{2} m_{2} K}^{J M P}$ is the parity adapted bend-rotation function. The latter was expressed as a linear superposition of two products of an associated Legendre function of $\theta_{1}$, a spherical harmonic function of $\left(\theta_{2}, \phi_{2}\right)$, and a Wigner function of the Euler angles $(\alpha, \beta, \gamma)$, with $P=0$ and $P=1$ corresponding to even and odd parity, respectively. The frame defining all coordinates and vectors used in the DSL calculations is shown in Figure 1b). The basis set parameters, which were found to provide an optimal compromise between accuracy and computational cost, are listed in Table I. This basis is called "basis I" in Ref. 7 and was shown to give levels up to $3000 \mathrm{~cm}^{-1}$ with errors less than $0.01 \mathrm{~cm}^{-1}$ for $\mathrm{Cl}^{-}\left(\mathrm{H}_{2} \mathrm{O}\right)$. The PODVR basis functions are defined by a cut reference potential with other coordinates fixed at their values at the $C_{2 v}$ saddle point. These coordinate values can be found in the Supporting Information. Using the $C_{2 v}$ saddle point rather than the $C_{s}$ equilibrium ensures that the stretch basis functions have symmetry when permuting the two $\mathrm{H}$ atoms of $\mathrm{H}_{2} \mathrm{O}$.

For comparison, the intramolecular vibrational frequencies of the water molecule within the $\mathrm{X}^{-}\left(\mathrm{H}_{2} \mathrm{O}\right)$ and $\mathrm{X}^{-}\left(\mathrm{D}_{2} \mathrm{O}\right)$ dimers were also calculated by combining the local-mode ${ }^{22,23}$ and local-monomer ${ }^{24}$ (LM) methods as described in Ref. 25. Within this LM scheme, the Hessian matrix at the optimized geometry of each dimer was calculated from finite-differentiation of analytic first derivatives of both TTM-nrg and MB-nrg PEFs. Local modes ${ }^{22,23,26}$ were 


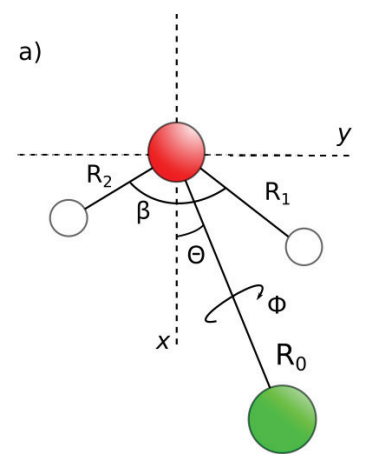

b)

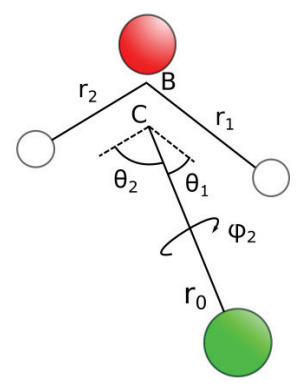

FIG. 1. a) Internal coordinate system for the halide-water dimers. X-axis is placed along the H-O-H angle, $\beta$, bisector. $R_{1}$ and $R_{2}$ are the two $\mathrm{OH}$ bond vectors. $R_{0}$ is the vector joining the oxygen atom and the halide ion. $(\Theta, \Phi)$ are the polar angles of $R_{0}$ in the coordinate system defined by the x-y axes. $\Theta$ is the same as $\theta_{\text {bis }}$ used to plot the PES in Ref. 7 b) Polyspherical coordinate system for the halide-water dimers based on Radau-Jacobi orthogonal vectors. $r_{1}$ and $r_{2}$ are Radau vectors pointing from the canonical point, labelled B. $\theta_{1}, \theta_{2}$ and $\phi_{2}$ are the polyspherical angles. $\mathrm{C}$ is the centre of mass of the water molecule.

TABLE I. Basis set parameters employed. The number of PODVR basis functions for each stretching coordinate given by $N_{r_{0}}, N_{r_{1}}$, and $\mathrm{N}_{r_{2}}$. Maximum values of $l$ and $m$ indices of the spherical harmonic basis, $l_{x}$ and $m_{x}$. The numbers of $\theta$ and $\phi$ quadrature points, $N_{\theta}$ and $N_{\phi}$. The number of even-parity basis functions, $N_{b a s}$.

\begin{tabular}{cc}
\hline \hline Parameter & Value \\
\hline$N_{r_{0}}$ & 24 \\
$N_{r_{1}}=N_{r_{2}}$ & 7 \\
$l_{x}=m_{x}$ & 25 \\
$N_{\theta}$ & 27 \\
$N_{\phi}$ & 54 \\
$N_{\text {bas }}$ & $7.29 \times 10^{6}$ \\
\hline \hline
\end{tabular}

then generated using a distance-based (Boys) localization criterion and frequency window of $500 \mathrm{~cm}^{-1}$ for both $\mathrm{X}^{-}\left(\mathrm{H}_{2} \mathrm{O}\right)$ and $\mathrm{X}^{-}\left(\mathrm{D}_{2} \mathrm{O}\right)$. The 3 highest-frequency (harmonic pseudofrequency) modes, corresponding to "left" and "right" single O-H (O-D) stretches and $\mathrm{H}-\mathrm{O}-\mathrm{H}$ (D-O-D) bends, were identified and used to integrate the potential energy using 
Gauss-Hermite quadrature on a grid of 9 points in each dimension. The local-monomer ${ }^{27,28}$ Hamiltonian was finally diagonalized to yield anharmonic frequencies and eigenvectors. Although this Hamiltonian was diagonalized in a reduced three-dimensional space for the water monomer, the potential energy was evaluated for the whole dimer, thereby including environmental effects. The expansion coefficients of the anharmonic wavefunction, in the harmonic basis, were used to assign bend overtones.

As mentioned above, two different PEFs (TTM-nrg ${ }^{13}$ and MB-nrg ${ }^{14}$ ) were used to represent the underlying PESs of $\mathrm{X}^{-}\left(\mathrm{H}_{2} \mathrm{O}\right)$ and $\mathrm{X}^{-}\left(\mathrm{D}_{2} \mathrm{O}\right)$ dimers with $\mathrm{X}=\mathrm{F}, \mathrm{Cl}, \mathrm{Br}$, and $\mathrm{I}$. In both PEFs, the intramolecular water distortion and the intermolecular water-water interactions are described by the many-body MB-pol PEF, ${ }^{29-31}$ which has been shown to predict the properties of water from the gas to the condensed phase with high accuracy. ${ }^{32-37}$ Both PEFs describe long-range ion-water interactions through permanent and induced electrostatics, within the Thole-type framework, ${ }^{38,39}$ and two-body dispersion energy. TTM-nrg and MB-nrg, however, differ in the way short-range interactions are represented. In TTM-nrg, the short-range interactions are modeled using Born-Mayer type functions of all interatomic distances. ${ }^{13}$ As discussed in Ref. 13, the Born-Mayer parameters were optimized by fitting each $\mathrm{X}^{-}\left(\mathrm{H}_{2} \mathrm{O}\right)$ TTM-nrg PEF to ab initio interaction energies calculated at the $\mathrm{CCSD}(\mathrm{T})$ F12 level of theory in the complete basis set (CBS) limit for $\sim 500$ dimer configurations. The MB-nrg PEFs, on the other hand, use permutationally invariant polynomials in transformed variables of all interatomic distances to accurately represent quantum-mechanical short-range effects (e.g., charge penetration, charge transfer, and Pauli repulsion) that become increasingly important when the electron densities of individual monomers (i.e., $\mathrm{X}^{-}$ and $\mathrm{H}_{2} \mathrm{O}$ ) overlap. The permutationally invariant polynomial of each MB-nrg PEF was originally trained on the interaction energies calculated at the CCSD(T)-F12/CBS level of theory on $\sim 12,000$ dissimilar $\mathrm{X}^{-}\left(\mathrm{H}_{2} \mathrm{O}\right)$ dimer configurations representative of the corresponding PES. ${ }^{14}$ The MB-nrg potentials were shown to accurately describe the corresponding halidewater dimer PESs and predict harmonic vibrational frequencies in close agreement with the corresponding CCSD(T)-F12/CBS reference data. ${ }^{14}$ Refined versions of the original permutationally invariant polynomials, in which both functional form and CCSD(T)-F12/CBS training sets were modified to improve the overall accuracy and stability of the MB-nrg PEFs, are used in this study. Specific details about all modifications are reported in the Supporting Information. 
It should be noted that no artificial constraints are applied, within the integration bounds of the internal coordinates, to either the TTM-nrg or the MB-nrg PEFs to obtain converged results in the present DSL calculations. As discussed in Refs. 13 and 14, both PEFs are derived from extensive training sets that effectively explore repulsive regions of the underlying PES and adopt functional forms where the combination of many-body classical electrostatics and short-range corrections provides a physically correct behavior at all internuclear separations. These two factors ensure that both PEFs are well behaved over a wide range of dimer configurations, including highly distorted (i.e., high potential energy) geometries. Ad hoc adjustments are commonly used to prevent the appearance of unphysical features on the calculated PESs (e.g., potential energy holes where the energy of a distorted molecular configuration is lower than the energy corresponding to the equilibrium geometry). Holes corresponding to configurations with short intermolecular distances and repulsive orienta-

tions were found on $\mathrm{X}^{-}\left(\mathrm{H}_{2} \mathrm{O}\right)$ PESs previously reported in the literature. ${ }^{7,10}$ Problematic regions were also found on the recent $\mathrm{F}^{-}\left(\mathrm{H}_{2} \mathrm{O}\right)$ PES, which were attributed to the opening of dissociation channels. ${ }^{6}$ Consequently, in the GENIUSH calculations of Ref. 6, the ranges of the coordinates for DVR functions were restricted to effectively avoid these problematic regions. The presence of holes on the PESs is particularly problematic when the Schrödinger equation is solved using a quadrature grid that spans the entire configuration space, as is the case in this study.

\section{RESULTS}

Due to the $C_{2 v}$ symmetry of the water molecule, the PES of each $\mathrm{X}^{-}\left(\mathrm{H}_{2} \mathrm{O}\right)$ dimer displays two equivalent global minima with $C_{s}$ symmetry which are separated by a $C_{2 v}$ transition state along the in-plane water rocking motion coordinate. Both location and height of the barrier on each PES depend on the strength of the corresponding hydrogen bond established by each halide ion and the water molecule. The minimum energy angular path along $\Theta$ as defined in Figure 1a), is obtained by doing a constrained optimization of all other coordinates keeping the $\Theta$ value fixed. It is shown in Figure 2 for all four halide-water PESs.

The barrier height systematically decreases from $\mathrm{F}^{-}\left(\mathrm{H}_{2} \mathrm{O}\right)$ to $\mathrm{I}^{-}\left(\mathrm{H}_{2} \mathrm{O}\right)$, reflecting the corresponding change in the nature of the underlying interactions, with $\mathrm{F}^{-}$exhibiting stronger and more covalent-like interactions with the water molecule due to its more pronounced ba- 

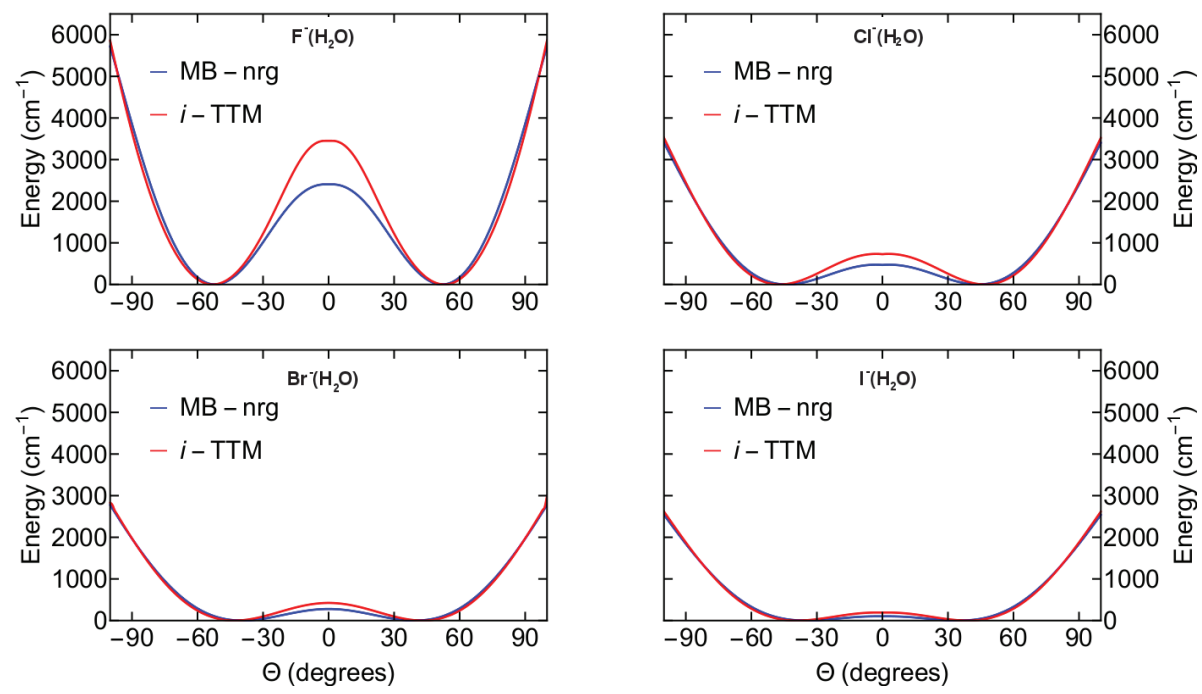

FIG. 2. Minimum energy path along the tunneling coordinate $\Theta$ (Figure 1a)), for the four halidewater dimers. See text for details.

sic character. As the ion size and associated polarizability increase the interactions within the $\mathrm{X}^{-}\left(\mathrm{H}_{2} \mathrm{O}\right)$ dimers become increasingly more electrostatic in nature. These changes in the underlying interactions are quantitatively predicted by the MB-nrg PEFs, which, as shown in Ref. 14, accurately reproduces $\operatorname{CCSD}(\mathrm{T})$-F12/CBS data for all dimer configurations. In contrast, as already discussed in previous studies, ${ }^{13,14}$ the TTM-nrg PEFs, as well as all other models that rely on a purely classical description of the intermolecular interactions, are not capable of correctly describing short-range interactions arising from the overlap of the monomer electron densities. These intrinsic deficiencies of the TTM-nrg PEFs are particularly evident in the comparisons with the corresponding MB-nrg results shown in Figure 2. For all four $\mathrm{X}^{-}\left(\mathrm{H}_{2} \mathrm{O}\right)$ dimers, the TTM-nrg PEFs always predict higher barriers, with the differences relative to the MB-nrg profiles becoming smaller as the classical electrostatic component of the interactions become larger (i.e., as both ion size and polarizability increase). The internal coordinates defining the positions of the global minimum $\left(C_{s}\right.$ configuration) and saddle point ( $C_{2 v}$ configuration) on the PESs described by both TTM-nrg and MB-nrg PEFs are listed in Table II for all four $\mathrm{X}^{-}\left(\mathrm{H}_{2} \mathrm{O}\right)$ dimers. The corresponding polyspherical coordinates based on the Radau-Jacobi orthogonal vectors are given in the Supporting Information.

To provide direct comparisons between the relative strength and anisotropy of the dif- 
ferent halide-water interactions, two-dimensional contour plots of the four dimer PESs are shown in Figure 3 as a function of the water $\mathrm{OH}$ stretching coordinate $R_{1}$ (on the $x$-axis) and the OX stretching coordinate $R_{0}$ (on the $y$-axis), with all other coordinates defined in Figure 1a) being kept fixed at the values corresponding to the minimum energy configurations (see Table II). As expected, the overall shape of the four PESs changes significantly depending on the nature of the interactions, with the minimum energy region moving to larger halide-water separations and systematically elongating along $R_{0}$ as the ion size increases. The more covalent-like nature of the $\mathrm{F}^{-}\left(\mathrm{H}_{2} \mathrm{O}\right)$ dimer becomes apparent in the relatively stronger attraction along $R_{1}$ for $R_{0} \sim 4.5$ bohr. Although both TTM-nrg and MB-nrg PEFs correctly predict the overall shape of all four $\mathrm{X}^{-}\left(\mathrm{H}_{2} \mathrm{O}\right)$ PESs, noticeable differences between the two sets of PEFs exist, which become progressively smaller from $\mathrm{F}^{-}\left(\mathrm{H}_{2} \mathrm{O}\right)$ to $\mathrm{I}^{-}\left(\mathrm{H}_{2} \mathrm{O}\right)$. In particular, the relatively larger differences between the TTM-nrg and MB-nrg PESs found for $\mathrm{F}^{-}\left(\mathrm{H}_{2} \mathrm{O}\right)$ reinforce the notion that purely classical PEFs, like TTM-nrg, are unable to describe quantitatively short-range interactions where quantum-mechanical effects associated with the overlap of the monomer electron densities (e.g., charge penetration and transfer, and Pauli repulsion) become increasingly important.

Further insights into the energy landscape associated with the halide-water dimers are obtained from the analysis of the two-dimensional contour plots of the four PESs shown in Figure 4 as a function of the angle $\Theta$ (on the $x$-axis) and OX stretching coordinate $R_{0}$ (on the $y$-axis), with all other internal coordinates (Figure 1a)) fixed at the values corresponding to the minimum energy configurations (Table II). All four contour plots display two minima corresponding to the two hydrogen-bonded configurations of the dimer. It is important to note that, since each contour plot is calculated keeping the internal coordinates $R_{1}, R_{2}$, and $\beta$ (Figure 1a)) of the water molecule fixed at the values of the corresponding minimum-energy dimer configuration, the two energy minima are not symmetric by construction. Figure 4 clearly shows that, going from $\mathrm{F}^{-}\left(\mathrm{H}_{2} \mathrm{O}\right)$ to $\mathrm{I}^{-}\left(\mathrm{H}_{2} \mathrm{O}\right)$, the two energy minima move to smaller $\Theta$ and become shallower.

Selected vibrational levels of the $\mathrm{X}^{-}\left(\mathrm{H}_{2} \mathrm{O}\right)$ and $\mathrm{X}^{-}\left(\mathrm{D}_{2} \mathrm{O}\right)$ dimers, calculated with the DSL method using both TTM-nrg and MB-nrg PEFs are listed in Tables III - VI. Along with the intramolecular water vibrational modes calculated with the LM method, as described previously. In the DSL calculations the vibrational states were identified by analyzing both probability densities and wavefunction amplitudes as functions of the coordinates of interest. 
FIG. 3. Two dimensional cuts of the TTM-nrg and MB-nrg PESs, for the four halide-water dimers, along two stretching coordinates $R_{1}$ and $R_{0}$ (Figure 1a)). The energy spacing between the adjacent lines is $500 \mathrm{~cm}^{-1}$. See text for details.

TTM-nrg

MB-nrg
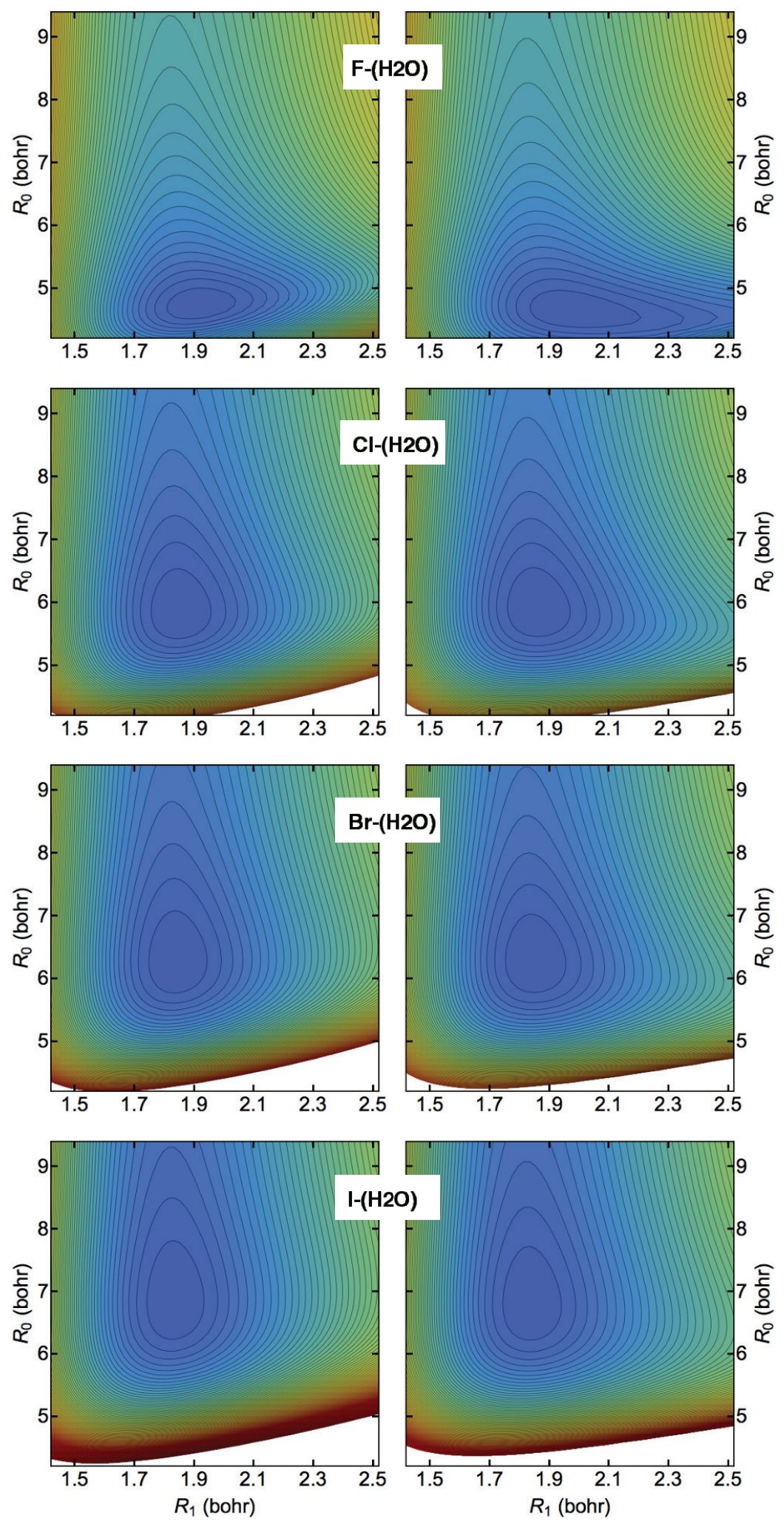
FIG. 4. Two dimensional cuts of the TTM-nrg and MB-nrg PESs, for the four halide-water dimers, along the tunneling coordinate, $\Theta$ and stretching coordinate, $R_{0}$ (Figure 1a)). The energy spacing between the adjacent lines is $500 \mathrm{~cm}^{-1}$. See text for details.

TTM-nrg
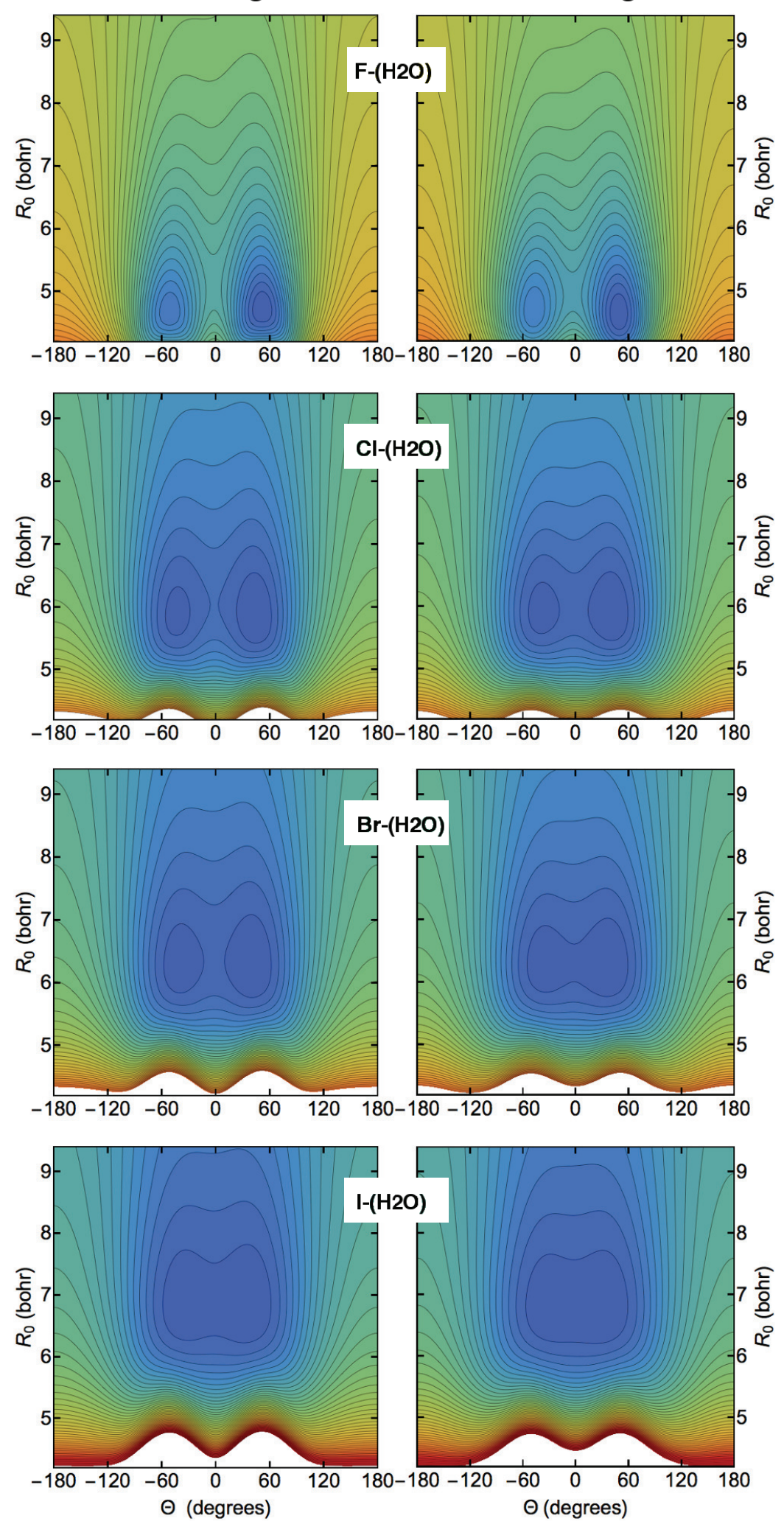
Plots of the probability densities along $\theta_{1}$ and $\theta_{2}$ (Figure 1b)) obtained with MB-nrg for the fundamental and the first overtone of the $\mathrm{HOH}$ bending vibration, the first overtone of the out-of-plane bending vibration, and the fundamental of the free $\mathrm{OH}$ stretching vibration of the $\mathrm{I}^{-}\left(\mathrm{H}_{2} \mathrm{O}\right)$ dimer are provided in the Supplementary Material as examples. It should be noted that, due to inter-mode couplings, some states, marked with asterisks in the tables cannot be unambiguously identified. Also included in the tables, as a reference, are the available experimental data. ${ }^{5,8,9}$

The DSL results with MB-nrg are in close agreement with the corresponding experimental values for all dimers, except for $\mathrm{F}^{-}\left(\mathrm{H}_{2} \mathrm{O}\right)$ for which the somewhat larger differences can be attributed to the covalent-like character of the fluoride-water interaction and the fact that, by construction, the MB-nrg PEF do not include the $\mathrm{HF}+\mathrm{OH}^{-}$dissociation channel for the dimer. The failure of the TTM-nrg PEFs to correctly describe short-range quantum-mechanical interactions manifest itself in vibrational frequencies that are noticeably blueshifted compared to the corresponding values obtained with the MB-nrg PEFs for the high frequency stretching modes. Importantly, the agreement with experimental values for the TTM-nrg PEFs improves from $\mathrm{F}^{-}\left(\mathrm{H}_{2} \mathrm{O}\right)$ to $\mathrm{I}^{-}\left(\mathrm{H}_{2} \mathrm{O}\right)$, reflecting the increase in classical-like nature of the interactions as the ion charge density decreases. This reinforces the notion that classical polarizable PEFs, being unable to accurately represent the intermolecular energy landscape, are not suited for quantitative studies of vibrational spectra, as previously suggested in Refs. 13 and 14. For all dimers, the LM results are in good agreement with the corresponding DSL results, except for $\mathrm{F}^{-}\left(\mathrm{H}_{2} \mathrm{O}\right)$ and $\mathrm{F}^{-}\left(\mathrm{D}_{2} \mathrm{O}\right)$ described by the MB-nrg PEF. These shortcomings can be explained by considering the complexity of the underlying PES as well as strong inter-mode couplings which make the LM approximations less accurate.

The three fundamental vibrational transitions (bend, symmetric and asymmetric stretches) of an isolated water molecule, as predicted by the Partridge-Schwenke water monomer $\mathrm{PES},{ }^{40}$ are $1594.78 \mathrm{~cm}^{-1}, 3657.04 \mathrm{~cm}^{-1}$ and $3755.96 \mathrm{~cm}^{-1}$ for $\mathrm{H}_{2} \mathrm{O}$ and, $1178.23 \mathrm{~cm}^{-1}$, $2671.33 \mathrm{~cm}^{-1}$ and $2787.59 \mathrm{~cm}^{-1}$ for $\mathrm{D}_{2} \mathrm{O}^{40}$ From the analysis of the vibrational frequencies of each halide-water dimer, it is evident that the vibrational dynamics of the water molecule is significantly affected by the presence of the halide anion. In the binary complexes, it is clearly not always valid to label strech vibrations as symmetric and asymmetric stretches, because both $\mathrm{OH}$ stretches become more localized. The hydrogen-bonded $\mathrm{OH}$ stretch is sig- 
nificantly redshifted, up to $\sim 1200 \mathrm{~cm}^{-1}$ in $\mathrm{F}^{-}\left(\mathrm{H}_{2} \mathrm{O}\right)$ due to the strong proton affinity of the fluoride anion, while the water free $\mathrm{OH}$ stretch vibrates at a much higher frequency, closer to that of the isolated water molecule. As expected, going from $\mathrm{F}^{-}\left(\mathrm{H}_{2} \mathrm{O}\right)$ to $\mathrm{I}^{-}\left(\mathrm{H}_{2} \mathrm{O}\right)$, the redshift of the hydrogen-bonded $\mathrm{OH}$ stretch decreases due to the weakening of the underlying intermolecular interactions. On the other hand, the water bend in the ion-water dimers is slightly blueshifted $\left(\sim 50 \mathrm{~cm}^{-1}\right.$ for the $\mathrm{X}^{-}\left(\mathrm{H}_{2} \mathrm{O}\right)$ dimers and $\sim 40 \mathrm{~cm}^{-1}$ for the $\mathrm{X}^{-}\left(\mathrm{D}_{2} \mathrm{O}\right)$ dimers) relative to the isolated water molecule since the bending motion becomes strained due to hydrogen bonding to the anion. For all vibrational states, the tunneling splitting increases going from $\mathrm{F}^{-}\left(\mathrm{H}_{2} \mathrm{O}\right)$ to $\mathrm{I}^{-}\left(\mathrm{H}_{2} \mathrm{O}\right)$, which can be explained by the lowering of the energy barrier between the two equivalent global minima along the tunneling coordinate $\Theta$ (Figure 1a)).

The DSL vibrational frequencies calculated with the TTM-nrg and MB-nrg PEFs are, in general, smaller than those obtained within both harmonic and one-dimensional anharmonic approximations for $\mathrm{X}^{-}\left(\mathrm{H}_{2} \mathrm{O}\right)$ dimers and their isotopologues (with $\mathrm{X}=\mathrm{Cl}, \mathrm{Br}$, and I). ${ }^{5}$ These differences, which are likely due to intrinsic limitations of the methods employed in Ref. 5, are particularly large for the more anharmonic modes involving the halide anions, such as the in-plane wag and hydrogen-bonded $\mathrm{OH}$ stretches.

As mentioned in the Introduction, among the halide-water dimers, $\mathrm{Cl}^{-}\left(\mathrm{H}_{2} \mathrm{O}\right)$ has been extensively studied using different theoretical approaches. The vibrational spectrum of $\mathrm{Cl}^{-}\left(\mathrm{H}_{2} \mathrm{O}\right)$ was obtained from both full-dimensional MULTIMODE ${ }^{10}$ and $\mathrm{DSL}^{7}$ calculations using a PES derived from high-level ab initio calculations. ${ }^{10}$ Good agreement is found between the results of Refs. 7 and 10 and the present DSL values obtained using both TTM-nrg and MB-nrg PEFs, with the frequencies reported here being consistently smaller for all vibrational modes. Considering that the present calculations were carried out with the same DSL method used in Ref. 7, these differences can be attributed to differences in the two PEFs used to represent the underlying $\mathrm{Cl}^{-}\left(\mathrm{H}_{2} \mathrm{O}\right)$ PES. Variational rovibrational energy levels of $\mathrm{F}^{-}\left(\mathrm{H}_{2} \mathrm{O}\right)$ and $\mathrm{F}^{-}\left(\mathrm{D}_{2} \mathrm{O}\right)$ dimers, along with the associated tunneling splittings, have also recently been calculated using a newly developed semiglobal PES. ${ }^{6}$ While close agreement is found between the vibrational frequencies reported in Ref. 6 and those obtained here with the MB-nrg PEF, the TTM-nrg values are significantly different, particularly for the stretching modes, due to already mentioned inaccuracies associated with the TTM-nrg description of short-range interactions within the fluoride-water dimer. To the best of our 
knowledge, no calculations of the vibrational spectra of $\mathrm{X}^{-}\left(\mathrm{H}_{2} \mathrm{O}\right)$ and $\mathrm{X}^{-}\left(\mathrm{D}_{2} \mathrm{O}\right)$ dimers with $\mathrm{X}=\mathrm{Br}$ and $\mathrm{I}$ have been reported in the literature.

\section{CONCLUSIONS}

A molecular-level understanding of halide-water interactions is key to characterizing the properties of complex electrolyte solutions that often mediate fundamental chemical, biological, and environmental processes. In this context, vibrational spectroscopy has emerged as a powerful tool to investigate structural and dynamical properties of aqueous systems from the gas phase to the condensed phase. The assignment of different spectral features is, however, complicated by the fluctuating nature of the underlying hydrogen-bond network, which often results in a continuum of distinct structural motifs that vary as a function of system size, thermodynamic conditions, and chemical environment. In this study, we have reported full-dimensional calculations of the anharmonic vibrational spectra of $\mathrm{X}^{-}\left(\mathrm{H}_{2} \mathrm{O}\right)$ and $\mathrm{X}^{-}\left(\mathrm{D}_{2} \mathrm{O}\right)$ dimers, with $\mathrm{X}=\mathrm{F}, \mathrm{Cl}, \mathrm{Br}$, and I, carried out using the DSL method with a product basis set including all six vibrational coordinates. The calculations have been performed on two different sets of many-body potential energy surfaces, TTM-nrg and MBnrg, which only differ in the way short-range interactions are represented. While within the TTM-nrg model, short-range interactions are modeled using common Born-Mayer functions, the MB-nrg PEFs use permutationally invariant polynomials in transformed variables of all interatomic distances to effectively represent quantum-mechanical short-range effects (e.g., charge penetration, charge transfer, and Pauli repulsion) that cannot be quantitatively described by classical expressions.

The present analysis demonstrates that the combination of state-of-the-art quantum methods with PEFs which, like MB-nrg, exhibit chemical accuracy enables the calculation of vibrational spectra in good agreement with experiment, correctly reproducing anharmonic and nuclear quantum effects. In contrast, the vibrational frequencies calculated with PEFs which, like TTM-nrg, represent many-body effects merely with a combination of classical electrostatics and two-body repulsion and dispersion contributions show significant devia-

tions from the corresponding experimental values. The comparison between the TTM-nrg and MB-nrg results thus indicates that an accurate and physically correct representation of both short- and long-range many-body interactions is necessary for the quantitative pre- 
diction of anharmonic vibrational frequencies of water molecules hydrogen-bonded to halide ions. Since the MB-nrg PEFs are fully transferable across different phases, future studies will focus on characterizing ion hydration from small clusters in the gas phase to bulk solutions and interfaces.

\section{SUPPLEMENTARY MATERIAL}

Details of the modifications made to the MB-nrg two-body PESs for halide-water systems. Polyspherical coordinates defining the positions of the global minimum $\left(C_{s}\right.$ configuration) and saddle point $\left(C_{2 v}\right.$ configuration) on the PESs described by both TTM-nrg and MBnrg PEFs for all four $\mathrm{X}^{-}\left(\mathrm{H}_{2} \mathrm{O}\right)$ dimers. Probability density plots along $\theta_{1}$ and $\theta_{2}$ for the fundamental and the first overtone of the $\mathrm{HOH}$ bending vibration, the first overtone of the out-of-plane bending vibration, and the fundamental of the free $\mathrm{OH}$ stretching vibration of the $\mathrm{I}^{-}\left(\mathrm{H}_{2} \mathrm{O}\right)$ dimer, obtained with MB-nrg. All vibrational levels below $3800 \mathrm{~cm}^{-1}$ of the $\mathrm{H}_{2} \mathrm{O}-\mathrm{X}^{-}$and $\mathrm{D}_{2} \mathrm{O}-\mathrm{X}^{-}, \mathrm{X}=\mathrm{F}, \mathrm{Cl}, \mathrm{Br}, \mathrm{I}$, dimers calculated using TTM-nrg and MB-nrg potentials.

\section{ACKNOWLEDGEMENTS}

We thank Prof. Mark Johnson for valuable discussions about experimental spectra of $\mathrm{I}^{-}\left(\mathrm{H}_{2} \mathrm{O}\right)$ and $\mathrm{I}^{-}\left(\mathrm{D}_{2} \mathrm{O}\right)$, and Prof. Ryan Steele and Dr. Xiaolu Cheng for their guidance in the LM calculations. We also thank Dr. Andreas Götz for guidance in the implementation of the TTM-nrg and MB-nrg PEFs in the RV4 code. This research was supported by the National Science Foundation Center for Chemical Innovation "Center for Aerosol Impacts on Climate and the Environment" (grant No. CHE-1305427) and the Natural Sciences and Engineering Research Council. Calculations were done using the Extreme Science and Engineering Discovery Environment (XSEDE), which is supported by the National Science Foundation (grant No. ACI-1053575, allocation TG-CHE110009) as well as the Triton Shared Computing Cluster (TSCC) at the San Diego Supercomputer Center. 


\section{REFERENCES}

${ }^{1}$ P. Ayotte, C. G. Bailey, G. H. Weddle, and M. A. Johnson, J. Phys. Chem. A 102, 3067 (1998).

${ }^{2}$ J. Kim, H. M. Lee, S. B. Suh, D. Majumdar, and K. S. Kim, J. Chem. Phys. 113, 5259 (2000).

${ }^{3}$ D. J. Tobias, P. Jungwirth, and M. Parrinello, J. Chem. Phys. 114, 7036 (2001).

${ }^{4}$ S. Yoo, Y. A. Lei, and X. C. Zeng, J. Chem. Phys. 119, 6083 (2003).

${ }^{5}$ S. Horvath, A. B. McCoy, B. M. Elliott, G. H. Weddle, J. R. Roscioli, and M. A. Johnson, J. Phys. Chem. A 114, 15561568 (2010).

${ }^{6}$ J. Sarka, D. Lauvergnat, V. Brites, A. G. Császár, and C. Léonard, Phys. Chem. Chem. Phys. 18, 17678 (2016).

${ }^{7}$ X.-G. Wang and T. Carrington Jr., J. Chem. Phys. 140, 204306 (2014).

${ }^{8}$ J. R. Roscioli, E. G. Diken, and M. A. Johnson, J. Phys. Chem. A 110, 4943 (2006).

${ }^{9}$ S. Horvath, A. B. McCoy, J. R. Roscioli, and M. A. Johnson, J. Phys. Chem. A 112, 1233712344 (2008).

${ }^{10}$ J. Rheinecker and J. M. Bowman, J. Chem. Phys. 125, 133206 (2006).

${ }^{11}$ X.-G. Wang and T. Carrington Jr., J. Chem. Phys. 117, 6923 (2002).

${ }^{12}$ X.-G. Wang, T. Carrington Jr., T. J., and A. R. W. McKellar, J. Chem. Phys. 123, 034301 (2005).

${ }^{13}$ D. J. Arismendi-Arrieta, M. Riera, P. Bajaj, R. Prosimiti, and F. Paesani, J. Phys. Chem. B 120, 1822 (2016).

${ }^{14}$ P. Bajaj, A. W. Götz, and F. Paesani, J. Chem. Theory Comput. 12, 26982705 (2016).

${ }^{15} \mathrm{X}$.-G. Wang and T. Carrington Jr., RV4 is a package of programs to compute rovibrational levels and wavefunctions of tetra-atomic molecules.

${ }^{16}$ J. Echave and D. C. Clary, Chem. Phys. Lett. 190, 225 (1992).

${ }^{17}$ H. Wei and T. Carrington Jr., J. Chem. Phys. 97, 3029 (1992).

${ }^{18}$ R. Chen and H. Guo, J. Chem. Phys. 114, 1467 (2001).

${ }^{19}$ X.-G. Wang and T. Carrington Jr., J. Chem. Phys. 114, 1473 (2001).

${ }^{20}$ M. J. Bramley and T. Carrington Jr., J. Chem. Phys. 99, 8519 (1993).

${ }^{21}$ U. Manthe and Köppel, J. Chem. Phys. 93, 345 (1990).

${ }^{22}$ X. Cheng and R. P. Steele, J. Chem. Phys. 141, 104105 (2014). 
${ }^{23}$ X. Cheng, J. J. Talbot, and R. P. Steele, J. Chem. Phys. 145, 124112 (2016).

${ }^{24}$ Y. Wang and J. M. Bowman, J. Chem. Phys. 134, 154510 (2011).

${ }^{25}$ S. E. Brown, A. W. Gtz, X. Cheng, R. P. Steele, V. A. Mandelshtam, and F. Paesani, J. Am. Chem. Soc. 139, 7082 (2017).

${ }^{26}$ C. R. Jacob and M. Reiher, J. Chem. Phys. 130, 084106 (2009).

${ }^{27}$ Y. Wang, B. C. Shepler, B. J. Braams, and J. M. Bowman, Chem. Phys. Lett. 491, 1 (2010).

${ }^{28}$ Y. Wang and J. M. Bowman, J. Phys. Chem. Lett. 4, 1104 (2013).

${ }^{29}$ V. Babin, C. Leforestier, and F. Paesani, J. Chem. Theory Comput. 9, 5395 (2013).

${ }^{30}$ V. Babin, G. R. Medders, and F. Paesani, J. Chem. Theory Comput. 10, 1599 (2014).

${ }^{31}$ G. R. Medders, V. Babin, and F. Paesani, J. Chem. Theory Comput. 19, 2906 (2014).

${ }^{32}$ G. R. Medders, A. W. Götz, M. A. Morales, P. Bajaj, and F. Paesani, J. Chem. Phys. 143, $104102(2015)$.

${ }^{33}$ G. R. Medders and F. Paesani, J. Chem. Theory Comput. 11, 1145 (2015).

${ }^{34}$ G. R. Medders and F. Paesani, J. Am. Chem. Soc. 138, 3912 (2016).

${ }^{35}$ F. Paesani, Acc. Chem. Res. 49, 1844 (2016).

${ }^{36}$ G. A. Cisneros, K. T. Wikfeldt, L. Ojamäe, J. Lu, Y. Xu, H. Torabifard, A. P. Bartók, G. Csányi, V. Molinero, and F. Paesani, Chem. Rev. 116, 7501 (2016).

${ }^{37}$ S. K. Reddy, S. C. Straight, P. Bajaj, H. C. Pham, M. Riera, D. R. Moberg, M. A. Morales, C. Knight, A. W. Götz, and F. Paesani, J. Chem. Phys. 145, 194504 (2016).

${ }^{38}$ B. T. Thole, Chem. Phys. 59, 341 (1981).

${ }^{39}$ P. T. v. Duijnen and M. Swart, J. Chem. Phys. A 102, 2399 (1998).

${ }^{40}$ H. Partridge and D. W. Schwenke, J. Chem. Phys. 106, 4618 (1997). 
TABLE II. Internal coordinates (see Figure 1a) for the $C_{s}$ minimum energy structure and the $C_{2 v}$ saddle point structure of the $\mathrm{X}^{-}\left(\mathrm{H}_{2} \mathrm{O}\right)$ dimers as predicted by the TTM-nrg and MB-nrg PESs. All structures are planar with $\Phi=180^{\circ}$. Distances $\left(R_{i}\right)$ in bohr, angles $(\Theta$ and $\beta)$ in degrees, and potential energy $(\mathrm{V})$ in $\mathrm{cm}^{-1}$.

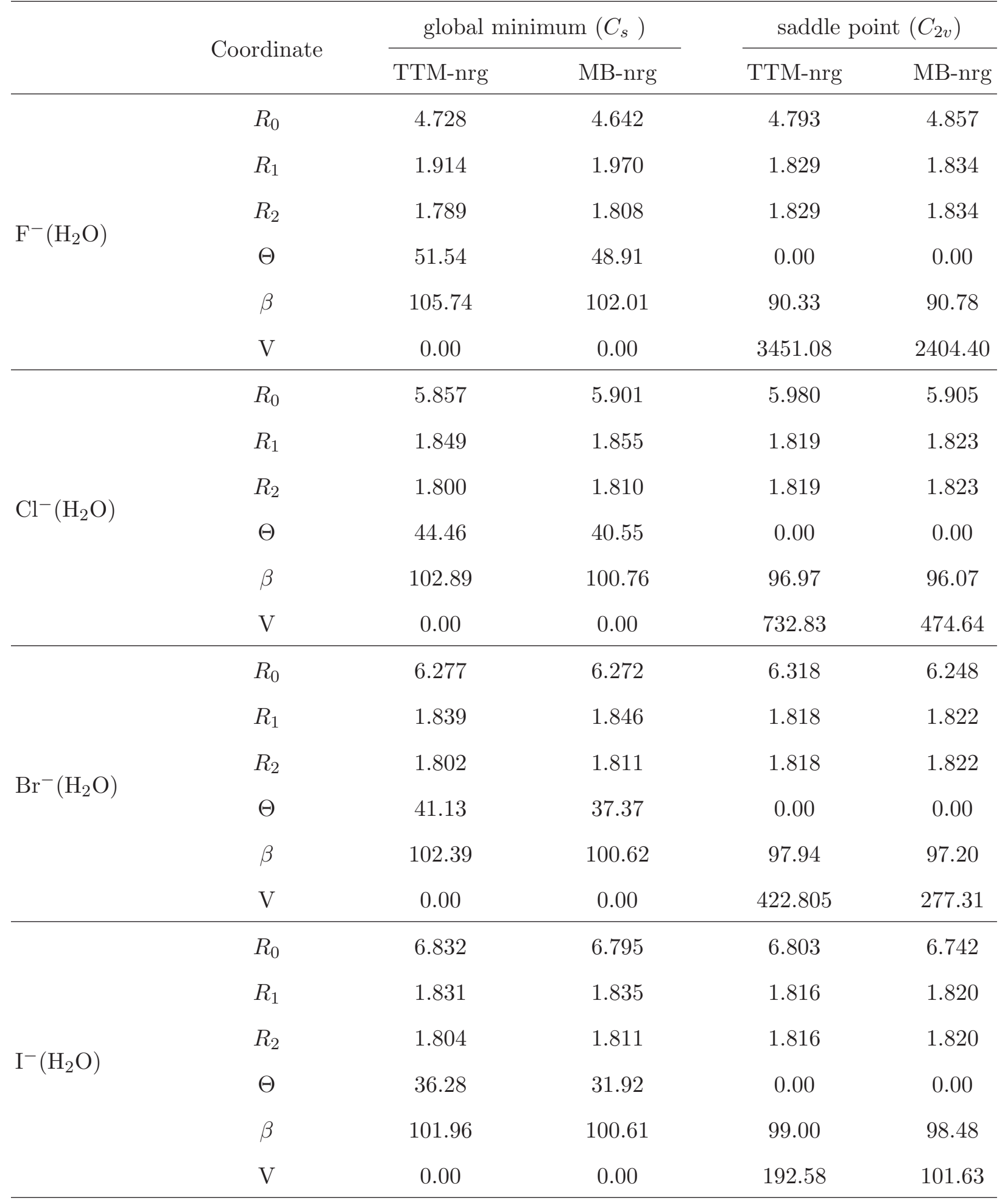


TABLE III. Selected vibrational levels of $\mathrm{F}^{-}\left(\mathrm{H}_{2} \mathrm{O}\right)$ (first row) and $\mathrm{F}^{-}\left(\mathrm{D}_{2} \mathrm{O}\right)$ (second row) obtained from DSL calculations with both TTM-nrg and MB-nrg PEFs. All values are in $\mathrm{cm}^{-1}$. The two tunneling-split partners, symmetric (ST) and anti-symmetric (AT), have even parity for all states expect for the out-of-plane bending mode fundamental which has odd parity. For comparison, the water intramolecular modes obtained within the LM approximation (see text for details) are shown in the table for both TTM-nrg and MB-nrg. Experimentally obtained frequencies are also included as a reference.

\begin{tabular}{|c|c|c|c|c|c|c|c|}
\hline \multirow{2}{*}{ Mode } & \multicolumn{3}{|c|}{ TTM-nrg } & \multicolumn{3}{|c|}{ MB-nrg } & \multirow{2}{*}{ Experiment } \\
\hline & $\mathrm{ST}$ & $\mathrm{AT}$ & $\mathrm{LM}$ & $\mathrm{ST}$ & AT & $\mathrm{LM}$ & \\
\hline \multirow[t]{2}{*}{$\mathrm{ZPE}$} & 5385.36 & 0.00 & & 5176.75 & 0.00 & & \\
\hline & 3973.60 & 0.00 & & 3695.05 & 0.00 & & \\
\hline \multirow[t]{2}{*}{$\nu_{\mathrm{FO}}$} & 435.74 & 435.74 & & 377.91 & 377.92 & & \\
\hline & 399.82 & 399.82 & & 368.58 & 368.58 & & \\
\hline \multirow[t]{2}{*}{$\nu_{\text {ip }}$} & 577.09 & 577.09 & & 556.74 & 556.75 & & \\
\hline & 453.82 & 453.82 & & 411.95 & 411.95 & & \\
\hline \multirow[t]{2}{*}{$\nu_{\mathrm{oop}}$} & 1021.91 & 1021.91 & & 1119.17 & 1119.17 & & \\
\hline & 733.88 & 733.88 & & 815.84 & 815.84 & & \\
\hline \multirow[t]{2}{*}{$2 \nu_{\mathrm{oop}}$} & 2429.97 & 2429.97 & & 2163.85 & 2164.03 & & \\
\hline & 1462.44 & 1462.44 & & 1582.14 & 1582.15 & & \\
\hline$\nu_{\mathrm{HOH}}$ & 1670.44 & 1670.44 & 1649.32 & $1748.34^{*}$ & $1748.36^{*}$ & 1675.96 & 1650 \\
\hline$\nu_{\mathrm{DOD}}$ & 1219.58 & 1219.58 & 1209.19 & 1258.01 & 1258.01 & 1394.58 & \\
\hline $2 \nu_{\mathrm{HOH}}$ & 3324.62 & 3324.77 & 3298.56 & & & 3321.87 & \\
\hline $2 \nu_{\mathrm{DOD}}$ & 2433.98 & 2433.99 & 2416.06 & 2535.16 & 2535.14 & 2740.40 & \\
\hline$\nu_{\text {boundOH }}$ & 3081.50 & 3081.47 & 3132.73 & $1435.86^{*}$ & $1435.86^{*}$ & 2109.81 & 1523 \\
\hline$\nu_{\text {boundOD }}$ & 2227.10 & 2227.10 & 2249.10 & $1076.44^{*}$ & $1076.44^{*}$ & 1576.15 & \\
\hline$\nu_{\text {freeOH }}$ & 3804.10 & 3804.10 & 3809.53 & $3729.84^{*}$ & $3730.42^{*}$ & 3782.53 & 3687 \\
\hline$\nu_{\text {freeOD }}$ & 2804.47 & 2804.47 & 2807.16 & 2839.46 & 2839.47 & 2963.79 & \\
\hline
\end{tabular}


TABLE IV. Selected vibrational levels of $\mathrm{Cl}^{-}\left(\mathrm{H}_{2} \mathrm{O}\right)$ (first row) and $\mathrm{Cl}^{-}\left(\mathrm{D}_{2} \mathrm{O}\right)$ (second row) obtained from DSL calculations with both TTM-nrg and MB-nrg PEFs. All values are in $\mathrm{cm}^{-1}$. The two tunneling-split partners, symmetric (ST) and anti-symmetric (AT), have even parity for all states expect for the out-of-plane bending mode fundamental which has odd parity. For comparison, the water intramolecular modes obtained within the LM approximation (see text for details) are shown in the table for both TTM-nrg and MB-nrg. Experimentally obtained frequencies are also included as a reference.

\begin{tabular}{|c|c|c|c|c|c|c|c|}
\hline \multirow{2}{*}{ Mode } & \multicolumn{3}{|c|}{ TTM-nrg } & \multicolumn{3}{|c|}{ MB-nrg } & \multirow{2}{*}{ Experiment } \\
\hline & $\mathrm{ST}$ & $\mathrm{AT}$ & $\mathrm{LM}$ & $\mathrm{ST}$ & AT & $\mathrm{LM}$ & \\
\hline \multirow{2}{*}{$\mathrm{ZPE}$} & 5152.60 & 0.23 & & 5065.90 & 1.04 & & \\
\hline & 3784.50 & 0.01 & & 3702.17 & 0.06 & & \\
\hline \multirow{2}{*}{$\nu_{\mathrm{ClO}}$} & 179.37 & 179.69 & & 185.64 & 186.86 & & \\
\hline & 174.62 & 174.63 & & 178.77 & 178.88 & & \\
\hline \multirow{2}{*}{$\nu_{\text {ip }}$} & 335.52 & 344.78 & & 287.13 & 316.60 & & 309 \\
\hline & 253.39 & 253.77 & & 223.77 & 226.80 & & 227 \\
\hline \multirow{2}{*}{$\nu_{\mathrm{oop}}$} & 703.00 & 702.41 & & 714.86 & 712.43 & & 738 \\
\hline & 511.61 & 511.60 & & 519.05 & 518.92 & & \\
\hline \multirow{2}{*}{$2 \nu_{\text {oop }}$} & 1367.51 & 1368.87 & & 1380.08 & 1385.05 & & 1404 \\
\hline & 1005.50 & 1005.56 & & 1015.68 & 1015.98 & & 1028 \\
\hline$\nu_{\mathrm{HOH}}$ & 1656.44 & 1656.96 & 1639.26 & 1674.70 & 1674.88 & 1624.49 & 1653 \\
\hline$\nu_{\mathrm{DOD}}$ & 1217.60 & 1217.63 & 1208.20 & 1216.68 & 1216.88 & 1201.00 & 1219 \\
\hline $2 \nu_{\mathrm{HOH}}$ & 3274.24 & 3274.33 & 3237.45 & 3315.02 & 3316.78 & 3227.44 & 3289 \\
\hline $2 \nu_{\mathrm{DOD}}$ & 2411.94 & 2412.04 & 2392.03 & 2442.25 & 2442.35 & 2393.42 & 2429 \\
\hline$\nu_{\text {boundOH }}$ & 3414.45 & 3414.70 & 3385.90 & & $3146.49^{*}$ & 3100.86 & 3146 \\
\hline$\nu_{\text {boundOD }}$ & 2503.72 & 2503.76 & 2487.66 & 2376.14 & 2376.16 & 2314.38 & 2341 \\
\hline$\nu_{\text {freeOH }}$ & 3754.30 & 3754.01 & 3757.96 & $3705.73^{*}$ & $3703.66^{*}$ & 3698.84 & 3697 \\
\hline$\nu_{\text {freeOD }}$ & 2766.47 & 2766.48 & 2770.96 & 2757.75 & 2757.79 & 2727.51 & 2737 \\
\hline
\end{tabular}


TABLE V. Selected vibrational levels of $\mathrm{Br}^{-}\left(\mathrm{H}_{2} \mathrm{O}\right)$ (first row) and $\mathrm{Br}^{-}\left(\mathrm{D}_{2} \mathrm{O}\right)$ (second row) obtained from DSL calculations with both TTM-nrg and MB-nrg PEFs. All values are in $\mathrm{cm}^{-1}$. The two tunneling-split partners, symmetric (ST) and anti-symmetric (AT), have even parity for all states expect for the out-of-plane bending mode fundamental which has odd parity. For comparison, the water intramolecular modes obtained within the LM approximation (see text for details) are shown in the table for both TTM-nrg and MB-nrg. Experimentally obtained frequencies are also included as a reference.

\begin{tabular}{|c|c|c|c|c|c|c|c|}
\hline \multirow{2}{*}{ Mode } & \multicolumn{3}{|c|}{ TTM-nrg } & \multicolumn{3}{|c|}{ MB-nrg } & \multirow{2}{*}{ Experiment } \\
\hline & $\mathrm{ST}$ & $\mathrm{AT}$ & LM & $\mathrm{ST}$ & $\mathrm{AT}$ & $\mathrm{LM}$ & \\
\hline \multirow{2}{*}{$\mathrm{ZPE}$} & 5142.86 & 0.22 & & 5022.77 & 5.63 & & \\
\hline & 3774.11 & 0.00 & & 3673.85 & 0.71 & & \\
\hline \multirow{2}{*}{$\nu_{\mathrm{BrO}}$} & 161.86 & 162.16 & & 146.67 & 153.19 & & \\
\hline & 156.26 & 156.27 & & 138.64 & 140.41 & & \\
\hline \multirow{2}{*}{$\nu_{\text {ip }}$} & 318.69 & 319.28 & & 209.21 & 281.71 & & \\
\hline & 252.93 & 253.28 & & 171.93 & 191.29 & & \\
\hline \multirow{2}{*}{$\nu_{\mathrm{oop}}$} & 703.07 & 698.46 & & 648.15 & 637.98 & & 664 \\
\hline & 511.34 & 511.33 & & 468.35 & 467.13 & & \\
\hline \multirow{2}{*}{$2 \nu_{\text {oop }}$} & 1367.77 & 1368.99 & & 1237.78 & 1255.52 & & 1262 \\
\hline & 1004.97 & 1005.03 & & 915.97 & 918.07 & & \\
\hline$\nu_{\mathrm{HOH}}$ & 1656.50 & 1656.96 & 1635.92 & 1666.76 & 1671.13 & 1619.04 & 1647 \\
\hline$\nu_{\mathrm{DOD}}$ & 1217.55 & & 1207.06 & 1212.99 & 1213.62 & 1197.06 & 1216 \\
\hline $2 \nu_{\mathrm{HOH}}$ & 3274.04 & 3274.15 & 3237.36 & 3316.99 & 3319.25 & 3197.13 & 3238 \\
\hline $2 \nu_{\mathrm{DOD}}$ & 2411.17 & 2411.24 & 2394.87 & 2407.20 & 2407.53 & 2370.85 & 2429 \\
\hline$\nu_{\text {freeOH }}$ & 3413.84 & 3414.10 & 3467.34 & $3303.80^{*}$ & $3297.91^{*}$ & 3268.14 & 3296 \\
\hline$\nu_{\text {freeOH }}$ & 2503.22 & 2503.23 & 2548.22 & 2474.93 & 2475.03 & 2429.17 & 2451 \\
\hline$\nu_{\text {freeOH }}$ & 3754.36 & 3754.10 & 3746.27 & $3697.55^{*}$ & $3693.17^{*}$ & 3698.40 & 3695 \\
\hline$\nu_{\text {freeOH }}$ & 2765.92 & 2765.90 & 2763.94 & 2764.93 & 2765.17 & 2728.10 & 2726 \\
\hline
\end{tabular}


TABLE VI. Selected vibrational levels of $\mathrm{I}^{-}\left(\mathrm{H}_{2} \mathrm{O}\right)$ (first row) and $\mathrm{I}^{-}\left(\mathrm{D}_{2} \mathrm{O}\right)$ (second row) obtained from DSL calculations with both TTM-nrg and MB-nrg PEFs. All values are in $\mathrm{cm}^{-1}$. The two tunneling-split partners, symmetric (ST) and anti-symmetric (AT), have even parity for all states expect for the out-of-plane bending mode fundamental which has odd parity. For comparison, the water intramolecular modes obtained within the LM approximation (see text for details) are shown in the table for both TTM-nrg and MB-nrg. Experimentally obtained frequencies are also included as a reference.

\begin{tabular}{|c|c|c|c|c|c|c|c|}
\hline \multirow{2}{*}{ Mode } & \multicolumn{3}{|c|}{ TTM-nrg } & \multicolumn{3}{|c|}{ MB-nrg } & \multirow{2}{*}{ Experiment } \\
\hline & $\mathrm{ST}$ & $\mathrm{AT}$ & $\mathrm{LM}$ & $\mathrm{ST}$ & AT & LM & \\
\hline \multirow{2}{*}{$\mathrm{ZPE}$} & 5032.21 & 16.37 & & 4965.06 & 27.05 & & \\
\hline & 3696.71 & 3.25 & & 3636.87 & 8.93 & & \\
\hline \multirow{2}{*}{$\nu_{\mathrm{IO}}$} & 110.39 & 128.89 & & 117.30 & 147.65 & & \\
\hline & 103.92 & 110.19 & & 97.62 & 121.72 & & \\
\hline \multirow{2}{*}{$\nu_{\text {ip }}$} & 166.17 & 237.89 & & 163.74 & 264.09 & & \\
\hline & 127.53 & 169.92 & & 122.00 & 171.83 & & \\
\hline \multirow{2}{*}{$\nu_{\mathrm{oop}}$} & 571.48 & 547.43 & & 591.24 & 554.28 & & \\
\hline & 408.89 & 404.22 & & 428.61 & 416.78 & & \\
\hline \multirow{2}{*}{$2 \nu_{\text {oop }}$} & 1065.37 & 1100.02 & & 1078.75 & 1128.13 & & 1098 \\
\hline & 891.66 & 900.64 & & 800.72 & 816.35 & & \\
\hline$\nu_{\mathrm{HOH}}$ & 1655.23 & 1667.77 & 1633.08 & 1657.81 & 1679.94 & 1614.00 & 1639 \\
\hline$\nu_{\mathrm{DOD}}$ & 1217.15 & 1219.53 & 1206.08 & 1208.39 & 1215.94 & 1193.91 & 1208 \\
\hline $2 \nu_{\mathrm{HOH}}$ & 3278.71 & 3287.33 & 3233.18 & 3305.16 & 3319.76 & 3197.13 & 3246 \\
\hline $2 \nu_{\mathrm{DOD}}$ & 2416.42 & 2418.02 & 2394.45 & 2404.39 & 2409.82 & 2370.85 & 2401 \\
\hline$\nu_{\text {boundOH }}$ & 3563.51 & 3566.31 & 3538.18 & $3395.84^{*}$ & $3422.35^{*}$ & 3398.83 & $3393 / 3422$ \\
\hline$\nu_{\text {boundOD }}$ & 2608.46 & 2609.41 & 2598.71 & 2550.55 & 2547.63 & 2515.35 & $2511 / 2527$ \\
\hline$\nu_{\text {freeOH }}$ & 3729.03 & 3730.23 & 3733.75 & $3696.56^{*}$ & 3702.08 & 3693.44 & $3692 / 3706$ \\
\hline$\nu_{\text {freeOD }}$ & 2751.61 & 2756.55 & 2757.17 & 2747.70 & 2740.96 & 2725.73 & 2728 \\
\hline
\end{tabular}

